\title{
Discussion on Strengthening and Repairing Methods of Masonry Structure
}

\author{
Baitao Sun ${ }^{\mathrm{a}}$, Guangzhi Liang ${ }^{\mathrm{b}, \text { * }}$ and Peilei Yan ${ }^{\mathrm{c}}$ \\ Key Laboratory of Earthquake Engineering and Engineering Vibration, Institute of Engineering \\ Mechanics, China Earthquake Administration, Harbin 150080, China \\ asunbt@iem.cn, bIgzstudy@126.com, cyanpeilei325@163.com
}

Keywords: Masonry structure, seismic reinforcement, repair.

Abstract. Masonry structure is the structure type that used most widely. However because of the structure characteristics and many other reasons, the seismic capacity of the masonry structure is relatively poor. It is greatly helpful to improve the seismic resistance of masonry structure by reinforcing structures before earthquake and repairing them after earthquake. This paper will sum up part of these methods for reference.

\section{Introduction}

Earthquake is one of the natural disasters that we often encounter. Earthquakes have always been to bring great distress to mankind for their unpredictability and enormous destructive. Especially with the development of economy and the increase of population density, earthquakes bring us more and more losses.

However, masonry structure is one of the most severely damaged structures in the earthquake. Masonry structure has some defects because of its structural characteristics. The integrity and ductility of masonry structures are poor, and tensile and shear strength are low. In all previous earthquakes, masonry structure often suffer the most serious damage, the earthquake damage data of multistory masonry buildings in Tangshan earthquake are shown in table $1 .^{[1]}$

Table 1 Statistics of Seismic Damage of Multistory Masonry Buildings in Tangshan Earthquake

\begin{tabular}{ccccc}
\hline Damage degree & \multicolumn{4}{c}{ Seismic intensity } \\
& 8 & 9 & 10 & 11 \\
\hline Basically intact & 11.3 & 1.3 & 0.6 & 0.3 \\
Slight damage & 35.3 & 6.8 & 5.0 & 1.5 \\
Moderate damage & 29.4 & 34.3 & 6.5 & 4.7 \\
Serious damage & 23.5 & 32.5 & 19.9 & 11.7 \\
Completely destroyed & 0 & 25.1 & 68.0 & 81.8 \\
\hline
\end{tabular}

Seismic reinforcement and repair are put on the agenda with more and more troubles from earthquakes. There is a close relationship between structural reinforcement and repair, but there are obvious differences between them. In simple terms, improving the original resistance of the structure, which we call it reinforcement; making the damaged structure to restore or exceed the original resistance, we call it structural repair. Usually when we call reinforcement, we need to improve its seismic level for some requirements refers to the structure in the intact state. However, when the structure suffers from the disaster and the bearing capacity of the structure has a certain degree of decline, we should restore the structure of the bearing capacity to ensure the structure function, and we call it repair. ${ }^{[2]}$ There is a distinct difference between the two from the origin.

\section{Characteristics of Masonry Structure}

Masonry structure refers to the structure that bears the main force of the building by walls and columns made by the block and mortar. It is divided into unreinforced masonry structure and reinforced masonry structure. Masonry structure has its own advantages. Masonry structure has such 
advantages as easy materials, low cost, no need of special tools when constructing, good fire resistance, and so on.

At the same time, there are still many problems in masonry structure. The brick wall is the main component of the multistory masonry building subjected to vertical load, and it is also the only component to bear the horizontal load. The brick wall prone to cross cracks caused by shear failure in the repeated action of seismic load. The masonry structure prone to brittle failure because of the poor integrity and low ductility, and it is known to enhance the integrity of masonry structure by ring beams and structural columns later. The force of structures will have a greater change when people change the requirements of building function. For the above reasons, we need to identify and reinforce structures for requirements and security of the buildings.

\section{Seismic Reinforcement of Masonry Structure}

Seismic strengthening is the design and construction for the existing buildings to meet the requirements of seismic identification. By reinforcement measures, we can improve the bearing capacity of the structure or component, enhance the integrity of masonry structure, strengthen the connection measures, ensure the original structure can work together with original structure, so that the structure can continue to use in the premise of security. The seismic reinforcement of multistory masonry buildings is to improve the seismic capacity of the structure by strengthening the components of the structure and change the way of stress for the structure. ${ }^{[3]}$ There are many different strengthening methods were used for different situations. Several methods of reinforcement are summarized here.

(1) Reinforcement with steel meshed cement mortar. The method makes the layer and the wall form a composite wall by adding a certain thickness of the cement mortar to the side of the column or the wall. The method belongs to a kind of composite interface strengthening method. The principle of the method is similar to structure member strengthening with strand steel wire web-polymer mortar and masonry strengthening with concrete splint. The construction sequence of reinforcement with steel meshed cement mortar are as follows: cleaning the original wall, drilling, cleaning, brushing the cement mortar, laying steel bars, installing anchor bars, watering the wall, plastering cement mortar layer by layer, curing after hardening, decoration construction. The key of this method lies in the connection between the layer and the original wall, so it is necessary to ensure the reliability of the reinforcement layer and the original wall. We can remove or repair the local wall if there is a serious damage on the wall. It's also suitable for a variety of situations.

Although the method has been more mature, there are still some shortcomings after analysis. This method was lack of relevant experimental data, and the experimental data was not comprehensive enough now. There was also a lack of experimental research on the fatigue performance and dynamic performance of the structure. And the existing research basically studied on the response forced one time, and did not consider the actual performance of the structure forced two times resulted from the reinforcement. This aspect of the study will be one of the trends in strengthening research.

(2) Structure member strengthening with carbonic fiber reinforced polymer. Improving the bearing capacity by using the structural adhesive to paste the fiber composite material on the surface of the wall, making full use of the excellent mechanical property of the fiber, to make the fiber work together with the member. The difficulty and emphasis of the method is how to paste the fiber material to the surface of the reinforcement member. The process of strengthening with fiber is as follows: cleaning the member surface, treatment on base surface before pasting, repairing the surface of component with special repair glue, bottom coating, pasting fiber, curing and coating. To prevent the distortion, separation and dislocation of fiber, we need control strictly the pasting position and lap length, also, the time interval between each process. It can greatly improve the bearing capacity and seismic capacity of the structure. And there are many other advantages such as good fatigue resistance, convenient construction, saving space, no effect on the quality and shape of the structure.

We can also find it that it can't improve the stiffness of the structure obviously, so the method has no obvious effect on the cracked structure. At the same time, there are some other deficiencies as high 
cost, poor fire resistance, no use in the case of insulation requirements and the brittleness after solidification of CFRP.

(3) The strengthening method by adding beam and reinforced concrete column. In view of the structural defects that masonry structure has the characteristic of poor integrity, improving seismic capacity with new ring beams and structural columns. Additional columns should have foundations, and be fully connected along the building. The column should be in the connection of the internal and external walls every one or two bays according to the fortification intensity and the number of layers in different locations of the building. Additional ring beams should connect tightly with the wall, to form a close system in the plane. ${ }^{[4]}$ We can take corresponding strengthening measures at the local transformation in elevation. This method can improve the integrity of the structure, and is suitable for other structures that ring beams and structural columns do not meet the requirements of the specification.

The biggest problem of the method is connection in present construction. There is a poor connection between the structure and structural columns. The current trend is to explore new construction methods to ensure the connection.

\section{Repair of Masonry Structure}

The damage of the structure was mainly shown in the form of cracks and deformations after the structure was subjected to the earthquake force. The repair of the cracks is the main direction of the repair after the earthquake. The main purpose of the masonry structure is to repair the damage of the structure and restore the bearing capacity of the structural members, so as to improve the bearing capacity, the integrity and the rigidity of the structure. The following is a summary of several repair methods.

(1) Pressure grouting. Grouting special glue, latex cement mortar or high strength non shrinkage grouting slurry into the cracks through the pressure in order to achieve the purpose of repairing cracks and restoring the integrity of the original structure and bearing capacity. The grouting method should be constructed in the following order: burying the grouting nozzle, sealing the seam, inspection of sealing, the configuration of grout, grouting, sealing and grouting quality inspection. The method is suitable for structures or members with smaller cracks, more cracks and no longer developed cracks. Current grouting technology has been relatively mature at present. It was popular for simple construction technology, rapid construction, short duration, and little effect from external construction conditions. But the scope of the method is limited.

(2) Seal filling repair. In the method, the 1:3 cement mortar, the cement mortar with a higher strength grade than the masonry mortar or the aggregate cement mortar mixed with 107 glue are filled into the brick joint to repair the cracks. The method can also be used with reinforcing bars. There are several points to pay attention to in this method. We must wait to construct on one side of the wall until the mortar has a certain strength on the other side of the wall. The two sides can't be on construction on the same crack at the same time. ${ }^{[5]}$ The method is suitable for the repair of the appearance of the wall and the structure with shallow cracks. The crack will be constrained greatly in this way, and the crack repair effect is good.

(3) Reinforced concrete pin-key. The method replaces the cracks with pin-keys, so as to restore the bearing capacity and integrity of the wall. Construction sequence is as follows, excavating vertical strips in the position of cracks, arranging the steel bars in the strip, using high strength non shrinkage grouting material to seal the strip. We need to clean the loose debris and dust after drilling and put the Steel cage into the stripe. We also should prepare tie bar with the high-strength cement mortar. ${ }^{[5]}$ The method is applicable to the horizontal cracks, cross cracks or one-way oblique cracks in the wall. This method can greatly improve the bearing capacity of the wall. 


\section{Conclusion}

Compared to the new house, seismic reinforcement and repair have significant advantages as low cost and less time. The masonry structure was widely used in society, especially in the industrial and civil buildings. Seismic reinforcement has a preventive effect, because the structure cannot meet the code requirements. The main cause of the repair is the structural damage caused by natural disasters such as earthquakes. There are many similarities between the two, but they can't be generalized.

Seismic reinforcement improves the bearing capacity and integrity of the structure, and there shouldn't be new adverse effects for the original structure in the premise of using requirements. Before selecting the reinforcement method, we should make seismic identification for the structure firstly. Seismic identification is the basis and criterion of seismic strengthening, and we can determine the requirements and parts according to the seismic identification. As for the structural repair, the building structure itself has already produced different degrees of damage. Structural resistance is reduced, or there are some hidden dangers in the building, resulting in cracks in the structure. It is necessary to analyze the cause of crack and its influence before taking measures.

In short, the choice of structural reinforcement and repair methods needs to be considered in many aspects, such as construction conditions, cost, duration and so on. Not only considering the safety of the structure, to achieve the basic design requirements, but also the comprehensive consideration of the economy and aesthetics, combined with the requirements of the actual situation and use, and then a comprehensive analysis of various factors, and finally we can choose suitable reinforcement methods.

At present, the system is more perfect and can provide a guarantee for the safety of the structure. The key is how to choose the appropriate reinforcement and repair method according to the actual situation. The selection of structural reinforcement and repair method is diverse. In response to the requirements of the national strategy of sustainable development, how to ensure the bearing capacity with the limited resources. New materials and methods will be the future development direction of reinforcement and repair.

\section{Acknowledgements}

This work were financially supported by the National Science \& Technology Pillar Program (2015BAK17B06), Seismic Industry Research Special fund under Grant No. 201508026 and Program for Innovative Research Team in China Earthquake Administration (Earthquake Disaster Simulation and Evaluation in mainland China).

\section{References}

[1] Zhao Yicheng, Zheng Yun. Study on Seismic Evaluation and Strengthening Method of Masonry Structure [C]. Editorial department of Chinese engineering mechanics. The seventeenth national conference on structural engineering papers. Wuhan: Engineering Mechanics magazine: 2008 III -465 .

[2] Zhu Bolong, Lu Zhoudao, Wu Gang. Disaster detection and strengthening of structures [M]. Shanghai: Tongji University press, 1995:44-46.

[3] People's Republic of China ministry of construction. (JGJ116-98) Technical code for seismic strengthening of buildings [S]. Beijing: China architecture and building press, 1998.

[4] Zhang Jingshu, Pan Baoyu. Current seismic strengthening methods and development trend [J]. Earthquake resistant engineering and strengthening, 2005, 27 (1): 57-61.

[5] Wang Fenglai. General analysis of earthquake damage of buildings and reinforcement of damaged buildings in Wenchuan earthquake [M]. Beijing: China Architecture and Building Press, 2009:7-13, 63-69. 